Gut, 1987, 28, S1, 95-101

\title{
Polyamines in intestinal and pancreatic adaptation
}

\author{
G D LUK, AND P YANG
}

Department of Medicine, Wayne State University School of Medicine, Detroit, Michigan, USA, Department of Medicine and The Oncology Center, The Johns Hopkins University School of Medicine, Baltimore, Maryland, USA

SUMMARY The intestinal mucosa is a rapidly proliferative tissue, with a highly dynamic cell population. Its total cellular mass is well controlled and can adapt, with hypo- or hyperplasia, to a wide variety of stimuli. Luminal nutrients, hormonal factors, and pancreatic and biliary secretions have all been implicated in the regulation of intestinal mucosal adaptation. Similarly, the same factors appear essential for the maintenance of exocrine pancreatic structure and function. The polyamines (putrescine, spermidine, and spermine) and the key enzyme controlling their synthesis (ornithine decarboxylase, ODC) are important for many cell growth processes, and may play important roles in intestinal and pancreatic adaptation. During intestinal adaptation in response to jejunectomy, lactation and pancreatico-biliary diversion (PBD), intestinal contents of ODC and polyamines are increased, paralleling increases in mucosal proliferative indices and DNA synthesis. With administration of the specific inhibitor of ODC (difluoromethylornithine, DFMO) the increases in ODC and polyamines are suppressed, and intestinal adaptation is abrogated. In pancreatic hyperplasia induced by caerulein, pancreatic polyamines are increased. With DFMO administration, caerulein-induced increases in pancreatic DNA synthesis were inhibited and pancreatic hypertrophy was partially suppressed. PBD-induced pancreatic hypertrophy, however, was not affected by DFMO. Thus, the role of polyamines in the adaptation of the pancreas, with a relatively quiescent proliferative status, is as yet undefined. It seems clear, however, that the induction of ODC and the resultant increase in polyamine biosynthesis are critical for the normal growth and especially for adaptive hyperplasia of the intestinal mucosa.

\section{Intestinal adaptation}

The intestinal mucosa is a highly proliferative tissue, and its rate of selfrenewal is such that it can completely replace its mass in three to six days. Intestinal mucosal proliferation is nevertheless highly regulated. Luminal nutrients, hormonal factors, and pancreaticobiliary secretions have all been implicated in the control of an intestinal mucosal proliferation. ${ }^{1}$

The mucosa can respond to situations where total absorptive surface is lost. After extensive small bowel resection in the rat, morphological and functional adaptation occur in the remaining intestine. This adaptation is greater in the distal intestine after proximal resection and is maximal near the anastomosis, tapering off distally. Intestinal adaptation is achieved by mucosal hyperplasia, which leads to increased villus height and crypt depth, as well as

Address for correspondence: Gordon D Luk, MD, Harper Hospital, GI, 3990 John R, Detroit, Michigan 48201, USA. dilation and lengthening of the intestinal remnant. This adaptive hyperplasia is dependent on a combination of factors, including intraluminal nutrients, ${ }^{23}$ pancreaticobiliary secretions ${ }^{45}$ and hormonal factors, most likely enteroglucagon. ${ }^{6}$

The mucosa can also respond to hyperphagia during lactation in the rat. ${ }^{7}$ During this process, intestinal adaptation with mucosal hyperplasia occurs, with increases in villus height and crypt depth, as well as increased glucose and water absorption per unit length of intestine. This morphological and functional adaptation is again greatest in the distal small intestine. Although luminal nutrients and hyperphagia may play a role, intestinal adaptation during lactation is most probably caused by hormonal factors. ${ }^{\text {? }}$

The contribution of luminal nutrients to mucosal growth (and pancreatic growth) was established by showing that exclusion of all luminal nutrients while maintaining total body nutrition by TPN resulted in mucosal and pancreatic hypoplasia. ${ }^{89}$ When chole- 
cystokinin (CCK) was added to the intravenous solution, pancreatic growth, but not intestinal growth, was stimulated. ${ }^{10}$ This confirmed the interrelationships between intestinal and pancreatic growth processes.

One of the most interesting models for intestinal adaptation, and one which allows the concurrent studies of intestinal and pancreatic adaptation, is that of pancreatico-biliary diversion (PBD), described by Dowling's laboratory. ${ }^{411}$ In this model, the proximal or distal half of the small bowel is interposed isoperistaltically between the pylorus and the ampulla of Vater, thus allowing one to dissect the potential role of pancreaticobiliary secretion. Using this model Dowling and his coworkers have been able to establish firmly the integration of the intestinal and pancreatic responses and the concept of an enteropancreatic trophic axis. Their data have shown that the pancreas exerts an indirect trophic effect on the intestinal mucosa and that the intestinal mucosa, through its endocrine secretion, controls in part the growth and function of the exocrine pancreas. ${ }^{4511}$

After PBD and jejunectomy, intestinal adaptive hyperplasia and pancreatic adaptive hyperplasia were observed. In comparing the PBD model to the jejunectomy model, it seemed clear that the PBD model induced both pancreatic and intestinal adaptation. Whereas, after jejunectomy, unless $90 \%$ of the small bowel was resected, adaptive hyperplasia of the pancreas was not stimulated to any appreciable extent. ${ }^{4511}$

Pancreatic hyperplasia could also be induced by the infusion of CCK or the CCK analogue caerulein. With pancreatic hypertrophy stimulated by caerulein, increases in the pancreatic weight were significantly correlated with rates of increases in pancreatic DNA content and DNA synthesis. ${ }^{12}$

Although the above evidence clearly shows the potential contribution of luminal nutrients, hormonal factors, and pancreaticobiliary secretions in the control of intestinal and pancreatic growth, the cellular mechanisms controlling these adaptive phenomena are poorly understood. The results of recent studies, ${ }^{13}$ particularly those from the laboratory of Dowling, ${ }^{14}$ suggest that the naturally occurring polyamines, putrescine, spermidine, and spermine, may play a critical role in regulating the adaptive changes in intestinal and pancreatic proliferation and growth.

\section{Ornithine decarboxylase and the polyamines}

The amino acid ornithine, unlike other amino acids, is not used to synthesise protein. It is not represented by any triplet codon in the genetic code and is not recognised by any specific tRNA. Ornithine, however, does participate in three separate metabolic pathways.
It is metabolised via the Krebs cycle through ornithine transaminase with resultant formation of carbon dioxide and water. Ornithine is also an intermediate in the urea cycle, being converted by ornithine transcarbamylase to citrulline, which is then converted in sequence to arginosuccinate, arginine, and urea. The third metabolic pathway that ornithine participates in and the subject of the present paper is the polyamine biosynthetic pathway. ${ }^{15-19}$

Ornithine is the starting substrate for the biosynthesis of the polyamines putrescine, spermidine, and spermine. It is decarboxylated by ornithine decarboxylase (ODC, EC 4.1.1.17) to form putrescine, and this reaction is the first and rate limiting step in polyamine biosynthesis. The second critical enzyme is S-adenosylmethionine decarboxylase (SDC, EC 4.1.1.50), which catalyses the decarboxylation of Sadenosyl methionine, thus providing propylamino groups for the subsequent biosynthesis of spermidine and spermine via spermidine and spermine synthesis. ${ }^{15-19}$ Diamine oxidase (DAO) is the putative enzyme for degradation of putrescine (Fig. 1).

The structure of the polyamines are as follows: putrescine: $\mathrm{NH}_{2}\left(\mathrm{CH}_{2}\right)_{4} \mathrm{NH}_{2}$; spermidine: $\mathrm{NH}_{2}\left(\mathrm{CH}_{2}\right)_{4}$ $\mathrm{NH}\left(\mathrm{CH}_{2}\right)_{3} \mathrm{NH}_{2} ;$ spermine: $\mathrm{NH}_{2}\left(\mathrm{CH}_{2}\right)_{3} \mathrm{NH}\left(\mathrm{CH}_{2}\right)_{4}$ $\mathrm{NH}\left(\mathrm{CH}_{2}\right)_{3} \mathrm{NH}_{2}$.

The polyamines are ubiquitous polycationic compounds synthesised by all nucleated prokaryotic and eukaryotic cells studied. They form non-covalent complexes with many organic molecules, especially the nucleic acids, and have been shown, in vitro, to facilitate nearly all aspects of DNA, RNA, and protein synthesis. They are also known growth factors for certain prokaryotic and eukaryotic cells studied. The basal activity of ornithine decarboxylase, the first and rate limiting enzyme in polyamine biosynthesis, is low in most tissues. But marked increases in enzyme activity and rapid accumulation of tissue polyamines are characteristically associated with rapid tissue and cell growth, as evidenced by studies of embryonic growth, tumour growth, and increased secretory activity of endocrine glands. This increase in ornithine decarboxylase activity, frequently stimulated by hormones in their target tissues, is one of the earliest events which occur during the transition of cells from dormancy to active proliferation..$^{15-19}$

Ornithine decarboxylase has, perhaps, the shortest half-life known for a mammalian enzyme, ranging from seven to 15 minutes in different reports. In the rat partial hepatectomy model, increased ornithine decarboxylase activity and increased RNA synthesis occur almost simultaneously. Other studies suggested that ornithine decarboxylase may be an initiation factor for RNA polymerase I, the enzyme responsible for rRNA synthesis. The dramatic early increase in ornithine decarboxylase activity in the initial phases of rapid cell proliferation is thus different from the 


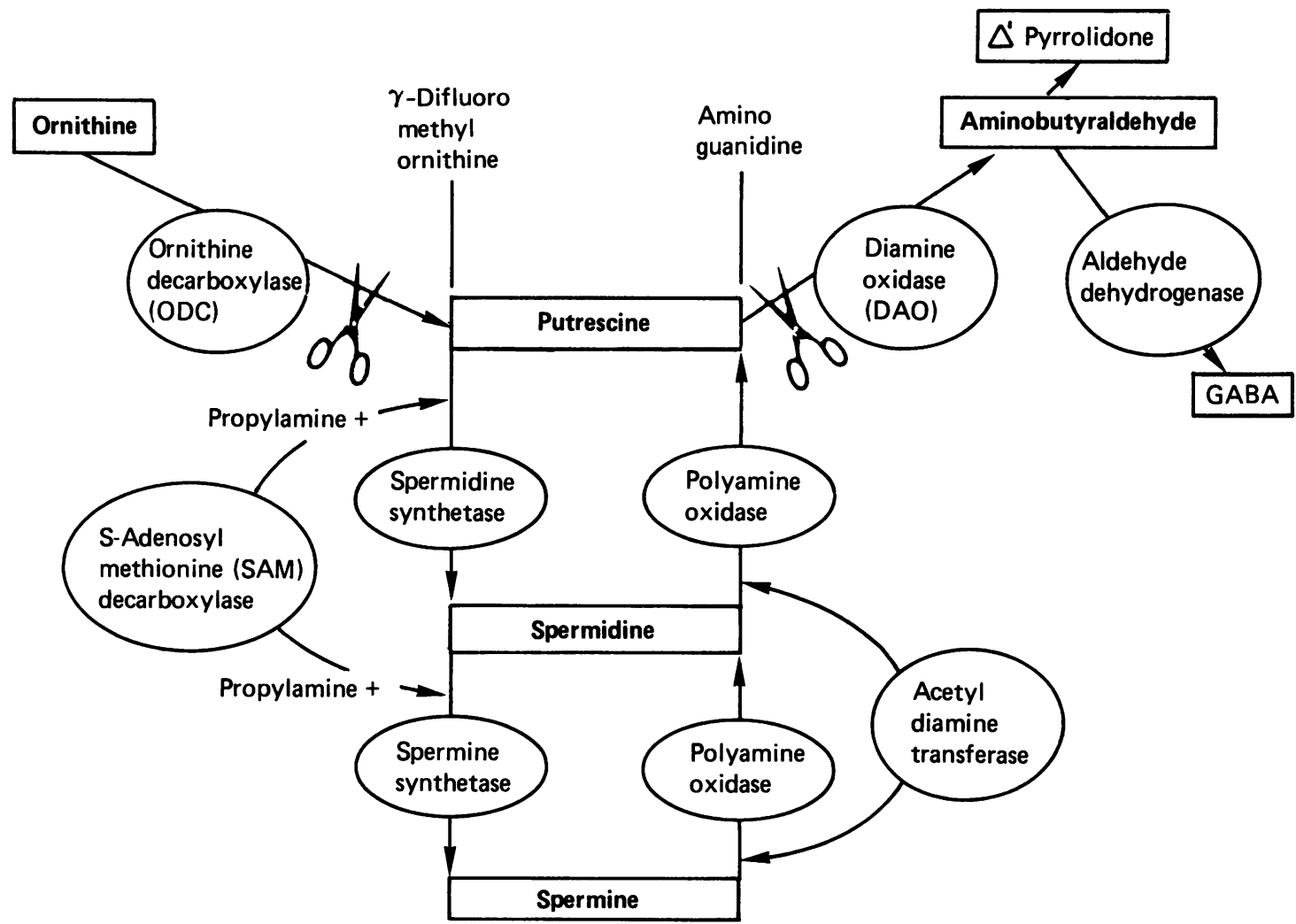

Fig 1. Schema of the biochemical pathways involved in the synthesis and degradation of the naturally-occurring polyamines, putrescine, spermidine, and spermine, from the amino acid ornithine. Arrows represent pathways, scissors represent inhibition/blockade. (Reproduced with permission from Dowling, RH, et al Scand J Gastroenterol 1985; 20 : suppl. 112: 84-95.

behaviour of most other mammalian enzymes. Ornithine decarboxylase seems poised for rapid and transient increases which could trigger critical processes in cell proliferation and protein synthesis. ${ }^{15-19}$

Studies using blockade of polyamine synthesis have provided important information about the potential roles of the polyamines. ${ }^{1819}$ Particularly important in this regard is the specific enzyme activated, irreversible ornithine decarboxylase inhibitor, difluoromethyl ornithine (DFMO, MDL 71, 782), synthesised at the Merrell-Dow Research Institute. ${ }^{20}$ This drug has made possible the sustained depletion of polyamines and allowed documentation of the critical role of the polyamines in several biologic processes. ${ }^{181921}$ DFMO has no acute pharmacologic activity other than the selective inhibition of ornithine decarboxylase, and is virtually non-toxic in normal mice and rats at doses sufficient for in vivo inhibition of tissue ornithine decarboxylase.

In vitro, DFMO has been shown to suppress the early increase in ornithine decarboxylase activity which accompanies the onset of proliferation of several cell types. This inhibition retards the growth of many cells in culture, including rat hepatoma cells, mouse mammary EMT6 sarcoma cells, mouse L1210 leukaemia cells, human prostate adenoma cells, and human small cell lung carcinoma cells. In vivo, DFMO suppresses the increase in uterine ornithine decarboxylase activity associated with early embryogenesis and arrests embryonic development. These results suggest that increases in ornithine decarboxylase activity and polyamine synthesis may play essential roles in mammalian cellular growth processes. ${ }^{1821}$

\section{Polyamines and intestinal and pancreatic adaptation}

Using the newborn rat intestinal maturation model, we initially found increases in mucosal polyamine content and ornithine decarboxylase activity during the time period of maximal intestinal mucosal growth, and increases in mature mucosal enzymes. ${ }^{22}$ As described in a subsequent section, administration of 
DFMO resulted in abrogation of the maturation pr scess. These early results suggested that the polyamines may indeed play a critical role in intestinal mucosal growth processes. ${ }^{22}$

Using an adaptation and modification of several established analytical methods, Dowling's laboratory was able to extensively quantify levels of the three naturally occurring polyamines, as well as cadaverine, throughout the entire length of the intestinal tract of the normal rat. Spermidine was present in the largest amount for each of the intestinal segments, followed by spermine, putrescine and cadaverine. ${ }^{23}$ There was a proximal-to distal gradient in the amounts of all the polyamines per length of intestine with proximal duodenal levels being greatest. This proximal-to distal gradient persisted even when the results were expressed as per milligram mucosal DNA. Furthermore, the spermidine/spermine ratio was also highest in the duodenum and lowest in the colon, and the ratio parallels the established pattern of cellular proliferation in normal rats tested, being highest in the duodenum and jejunum. ${ }^{23}$

During mucosal adaptive hyperplastic growth, polyamine content and ODC, when measured, has been found to increase in a manner paralleling the increase in proliferative indices. Specifically, we have shown that mucosal ODC and polyamines are increased during intestinal maturation, ${ }^{22}$ intestinal mucosal recovery after injury with cytosine-arabinoside, ${ }^{22}$ and during adaptive hyperplasia after jejunectomy ${ }^{24}$ and during lactation. ${ }^{25}$

Using the PBD model, Dowling and his coworkers were also able to show marked increases in mucosal polyamine levels with intestinal adaptive hyperplasia after PBD. ${ }^{1426}$ They showed that the relative increases in polyamine in the ileum were greater than those in the jejunum which, in turn, were greater than those in the duodenum, paralleling the degree of adaptive response in the various intestinal segments. The percentage increases were greatest with spermidine, followed by spermine, and putrescine. ${ }^{26}$ They found similar increases in polyamines with adaptive intestinal hyperplasia after jejunectomy. In correlating the increases in polyamine levels with the levels of circulating peptide hormones, they found that enteroglucagon was possibly the strongest candidate for the trophic hormone associated with intestinal hyperplastic growth. ${ }^{27}$

Similarly, changes in pancreatic polyamine levels were found with pancreatic hyperplastic growth. ${ }^{28}$ Using the caerulein model of pancreatic hypertrophy, increases in all three naturally occurring polyamines were seen within 12-96 hours after the beginning of caerulein administration. This time period coincided with pancreatic hypertrophy and hyperplasia as measured by increased cell mass and DNA content.
The total spermidine and spermine content were also significantly correlated with the rates of pancreatic weight and DNA content increases. ${ }^{28}$

Although these strongly positive correlations between the polyamines and intestinal mucosal and pancreatic growth are highly suggestive of a role for the polyamines in intestinal and pancreatic adaptation, they are by no means conclusive. One potential troubling finding is that of the villus crypt distribution of ODC and the polyamines. Several laboratories, including ours, have found that ODC and putrescine are found in much higher concentrations in the mature, non-dividing villus tip cells than in the proliferating crypt cells. ${ }^{293031}$ In more recent studies, it was found that S-adenosylmethionine decarboxylase, and spermidine and spermine, do show the expected distribution of being higher in the proliferating crypt cells than in the non-dividing senescent villus tip cells. ${ }^{31}$

Despite these potentially troubling findings in the crypt-villus distribution of ODC and putrescine, studies using the specific ODC inhibitor, DFMO, should allow us to further dissect the potential role of polyamines and ODC in intestinal and pancreatic adaptive growth processes.

\section{Difluoromethylornithine inhibition of intestinal and pancreatic adaptation}

As described briefly above, the administration of DFMO resulted in suppression of increases in ODC activity and polyamine content, and resulted in delay and inhibition of intestinal maturation. ${ }^{22}$ This suggested that the increase in ODC and polyamines were important for the mucosal growth processes ongoing during intestinal maturation.

Another finding seen during some of these initial studies was that DFMO also reduced the food intake and body weight of rodents, especially when given over a prolonged period of time. ${ }^{2242532}$ In order to clearly show that the mucosal growth inhibitory effects of DFMO were due to specific ODC inhibition are not due to the accompanying weight loss, a pair feeding regimen should be used in most studies of DFMO where the drug is used for greater than three to seven days. ${ }^{32}{ }^{33}$ Parenthetically, when aminoguanidine, the inhibitor of the enzyme diamine oxidase, the putative degradative enzyme for putrescine was given, there is an increase in food intake and weight gain in rodents (Luk, unpublished observation). This suggests that manipulation of the polyamine pathway is possible both through the synthetic pathway (DFMO inhibition of ODC) and the degradative pathway (aminoguanidine inhibition of diamine oxidase).

Using the model of jejunectomy induced intestinal 


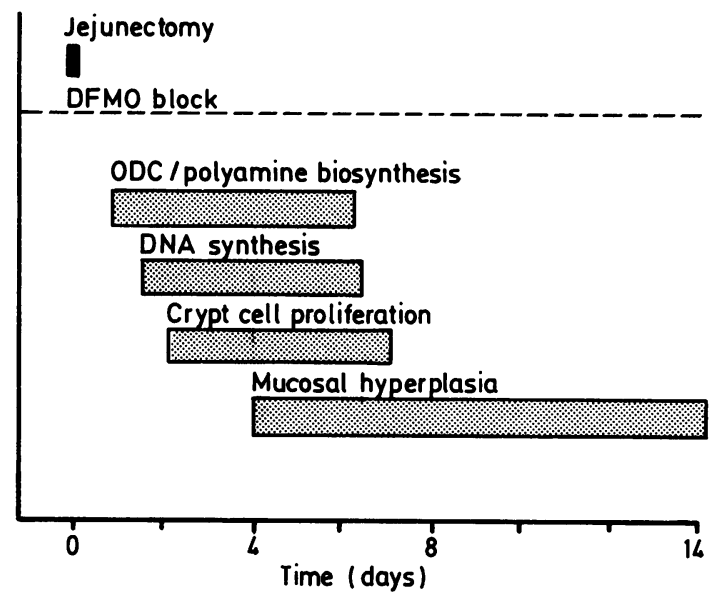

Fig 2. Schema of the sequence of cellular events leading to intestinal mucosal adaptation after jejunectomy. The individual cellular event is shown for that time period during which at least $25 \%$ of the total increase has occurred. The schema for the time course of cellular events thus indicates when each parameter depicted is substantially increased from basal, control levels. ODC/polyamine biosynthesis represents the increase in $O D C$ activity and polyamine content, DNA synthesis, the increase in DNA content and DNA specific activity, crypt cell proliferation, the increase in CCPR, and mucosal hyperplasia, the increase in mucosal height and weight. The dotted line for DFMO block depicts the complete suppression by DFMO of all cellular events leading to mucosal adaptation.

adaptive hyperplasia, we were able to show that DFMO could significantly inhibit the associated increases in ODC activity and polyamine biosynthesis. ${ }^{13}$ This biochemical effect of DFMO was associated with a marked inhibition of mucosal adaptive growth, in parallel with inhibition of increases in DNA synthesis, DNA content, and mucosal proliferative indices including crypt cell production rate. ${ }^{13}$ The sequence of biochemical and biological events could be clearly dissected in this model (Fig. 2).

Similarly, using the lactation-hyperphagia model of intestinal adaptation, we were able to show that the administration of DFMO resulted in significant inhibition of the increases in ODC and polyamines, and resulted in marked inhibition of the adaptive mucosal hyperplasia, in parallel with marked decreases in mucosal proliferative indices. ${ }^{25}$

Dowling and his coworkers extended this work to the PBD model. They showed that DFMO resulted in significant reduction in intestinal spermidine and spermine levels, and the spermidine/spermine ratio. This was associated with a marked reduction or complete prevention of the adaptive mucosal hyperplasia in both the jejunum and the ileum. However, the interpretation of these results might be hampered by the fact that a pair feeding regimen was not used, and the DFMO animals had significant weight loss compared with the control PBD animals. ${ }^{34}$

Using the same model, Dowling and his coworkers found that DFMO treated animals showed no differences in the amount of putrescine, spermidine or spermine in the whole pancreas, and that there were no differences in the mean value for wet weight protein or DNA between the untreated and DFMOtreated groups. They concluded that DFMO had no effect on the adaptive pancreatic hyperplasia induced by PBD. ${ }^{35}$ Again, interpretation of these data were difficult because of the marked weight loss experienced by the DFMO animals and the absence of a pair-fed control group. In contrast with the intestinal mucosa, however, cell proliferation of the pancreas is relatively quiescent, and mitotic figures in the normal pancreas are rare. Furthermore, the normal pancreas has very low basal tissue levels of ODC, and could be resistant to the effects of DFMO. ${ }^{14}$ Thus it would appear that pancreatic hyperplasia is much less dependent on ODC and polyamines than the intestinal mucosa.

DFMO alone did not affect the pancreatic gland at all. In the caerulein induced pancreatic hypertrophy model, DFMO reduced the increases in DNA synthesis and DNA content. DFMO also significantly suppressed the increases in pancreatic weight induced by caerulein ${ }^{36}$ In addition, this DFMO inhibition of pancreatic hypertrophy could be reversed by putrescine, a biosynthetic product of ODC. ${ }^{37}$ These studies are difficult to interpret because concurrent measurements of ODC or the polyamines were not provided. Taken together with the data in Dowling's PBD model of pancreatic hyperplasia, the conclusive proof that ODC and polyamines are important in pancreatic hypertrophy is not established.

In their studies of DFMO in the PBD model, Dowling and his coworkers also measured changes on other organs, kidneys, testes, and liver. They observed no changes in the fresh wet weight of kidneys and testes in the DFMO treated PBD animals. ${ }^{35}$ DFMO treated animals, however, show a marked reduction in whole liver weight, when compared with untreated PBD controls. Again, this was difficult to interpret because the DFMO animals had such marked weight reduction. ${ }^{3435}$ Using the posthepatectomy liver regeneration model, associated increases in ODC activity, polyamine content, and DNA and RNA syntheses, have been observed. ${ }^{38}$ Our laboratory showed recently that the administration of DFMO significantly inhibited the increases in ODC and polyamines associated with hepatic regeneration, and resulted in inhibition of hepatic DNA synthesis, proliferation, and liver weight gain. ${ }^{32}$ This DFMO 
inhibition of hepatic regeneration was reversed by the administration of putrescine..$^{32}$

The adaptive growth of other parts of the intestinal tract, in response to trophic stimuli, has also been shown to be regulated, at least in part, by the polyamines. Pentagastrin was shown to increase the total RNA, DNA, and protein content in the oxyntic gland of the gastric and duodenal mucosa of the rat. Concurrent treatment with DFMO completely inhibited the trophic response to pentagastrin in the oxyntic gland area. ${ }^{39}$ These preliminary data suggested that the trophic response of the oxyntic gland mucosa to stimulation by pentagastrin may require polyamine biosynthesis.

\section{Conclusion}

There appears to be extensive evidence to support the critical role of ODC and the polyamines in intestinal adaptation. The evidence supporting the role of ODC and polyamines in adaptation of the pancreas is less definitive. The use of pair feeding regimens, ${ }^{3233}$ pretreatment with DFMO to deplete the target organ of polyamines, ${ }^{3233}$ and use of long term cyclic regimens of DFMO administration ${ }^{33}$ may be possible avenues for further study.

The area of polyamine research has grown rapidly, and this includes the study of polyamines and intestinal and pancreatic adaptation. ${ }^{14}$ New and more potent inhibitors of polyamine biosynthesis, including more potent inhibitors of ODC, and newer polyamine analogues, have recently been described. ${ }^{21}$ In preliminary data in in vitro studies, the evidence suggests that the second generation inhibitors may allow one to achieve a more complete inhibition of ODC and/or a more complete depletion of tissue polyamines. ${ }^{21}$ They may allow one to clearly identify the role of ODC and polyamines in tissue growth processes. This increased availability of potential tools to study intestinal and pancreatic adaptation will lead to a better understanding of intestinal growth and function. This increased knowledge about the biology of intestinal function will be important for patients with malnutrition, secondary to the malabsorption caused by intestinal mucosal disease or resection, or to maldigestion because of pancreatic insufficiency.

We thank Drs Albert H Owens, Jr, Stephen B Baylin, and Thomas R Hendrix for encouragement, advice and support; Jeffrey Smith and William Theiss for technical assistance. The studies were supported in part by Grants R01-CA34453, R01-CA37789, and P01-CA37606 from the National Institutes of Health. GDL was a recipient of a faculty research award from the American Cancer Society and the
American Gastroenterological Association/Robbins Research Scholar Award.

\section{References}

1 Williamson RCN. Intestinal adaptation. $N$ Engl J Med 1978; 298: 1393-402, 1444-50.

2 Dowling RH. The influence of luminal nutrition on intestinal adaptation after small bowel resection and by-pass. In: Dowling RH, Riecken E-O, eds. Intestinal adaptation. Schattauer-Verlag, Stuttgart: $1974 ; 35-45$.

3 Morin CL, Grey VL, Garofalo C. Influence of lipids on intestinal adaptation after resection. In: Robinson JWL, Dowling RH, Riecken E-O, eds. Mechanisms of intestinal adaptation. Lancaster: MTP Press, 1982; 175-84.

4 Miazza BM, Hung L, Vaja S, Dowling RH. Effect of pancreatico-biliary diversion (PBD) on jejunal and ileal structure and function in the rat. In: Robinson JWL, Dowling RH, Riecken E-O, eds. Mechanisms of intestinal adaptation. Lancaster: MTP Press, 1982; 467-76.

5 Miazza BM, Turberg Y, Guillaume P, et al. Mechanism of pancreatic growth induced by pancreatico-biliary diversion in the rat. Inhibition by proglumide, benzotript, and ranitidine. Scand J Gastroenterol 1985; 20: suppl. 112: 75-83.

6 Bloom SR, Polak JM. Enteroglucagon and the gut hormone profile of intestinal adaptation. In: Robinson JWL, Dowling RH, Riecken E-O, eds. Mechanisms of intestinal adaptation. Lancaster: MTP Press, 1982; 17584.

7 Elias E, Dowling RH. The mechanism for small-bowel adaptation in lactating rats. Clin Sci Mol Med 1976; 51: 427-33.

8 Hughes CA, Prince A, Dowling RH. Speed of change in pancreatic mass and in intestinal bacteriology of parenterally-fed rats. Clin Sci 1980; 59: 329-36.

9 Fine H, Levine GM, Shiau Y-F. Effects of cholecystokinin and secretin on intestinal structure and function. Am J Physiol 1983; 245: G358-63.

10 Hughes CA, Breuer RS, Hatoff DE, Ducker DA, Dowling RH. The effect of CCK octapeptide on the pancreas: a study in rats during total parenteral nutrition (TPN) and oral feeding. [Abstract]. Eur J Clin Invest $1980 ; 10: 16$.

11 Miazza BM, Hung L, Vaja S, Dowling RH. Pancreatic hyperplasia after pancreatico-biliary diversion (PBD) in orally- and parenterally-fed rats. In: Robinson JWL, Dowling RH, Riecken E-O, eds. Mechanisms of intestinal adaptation. Lancaster: MTP Press, 1982: 481-90.

12 Solomon TE, Vanier M, Morisset J. Cell site and time course of DNA synthesis in pancreas after caerulein and secretin. Am J Physiol 1983; 8: G99-105.

13 Luk GD, Baylin SB. Inhibition of intestinal epithelial DNA synthesis and adaptive hyperplasia after jejunectomy in the rat by suppression of polyamine biosynthesis. J Clin Invest 1984; 74: 698-704.

14 Dowling RH, Hosomi M, Stace NH, et al. Hormones and polyamines in intestinal and pancreatic adaptation. Scand J Gastroenterol 1985; 20: suppl. 112: 84-95.

15 Russell DH, Durie BGM. Polyamines as biochemical 
markers of normal and neoplastic growth. New York: Raven Press, 1978.

16 Jänne JH, Pösö H, Raina A. Polyamines in rapid growth and cancer. Biochim Biophys Acta, 1978; 473: 241-93.

17 Williams-Ashman HG, Canellakis ZN. Polyamines in mammalian biology and medicine. Perspect Biol Med 1979; 22: 421-53.

18 Pegg AE, McCann PP. Polyamine metabolism and function. Am J Physiol 1982; 243: C212-21.

19 Tabor CW, Tabor H. Polyamines. Annu Rev Biochem 1984; 53: 749-90.

20 Metcalf BW, Bey P, Danzin C, et al. Catalytic irreversible inhibition of mammalian ornithine decarboxylase by substrate and product analogues. J Am Chem Soc 1978; 100: 2551-3.

21 Sjoerdsma A, Schechter P. Chemotherapeutic implications of polyamine biosynthesis inhibition. Clin Pharmacol Ther 1984; 35: 287-300.

22 Luk GD, Marton, LJ, Baylin SB. Ornithine decarboxylase is important in intestinal mucosal maturation and recovery from injury in rats. Science $1980 ; 210$ : 195-8.

23 Hosomi M, Smith SM, Murphy GM, Dowling RH. Polyamine distribution in the rat intestinal mucosa. $J$ Chromatogr 1986; 375: 267-75.

24 Luk GD, Baylin SB. Polyamines and intestinal growth increased polyamine biosynthesis after jejunectomy. Am J Physiol 1983; 245: G656-60.

25 Yang P, Baylin SB, Luk GD. Polyamines and intestinal growth: absolute requirement for ODC activity in adaptation during lactation. Am J Physiol 1984; 247: G553-7.

26 Miazza BM, Levan H, Vaja S, Dowling RH. Effect of pancreatico-biliary diversion (PBD) on the small intestine in orally and parenterally-fed rats. [Abstract]. Clin Sci 1981; 60: 6P.

27 Miazza BM, Levan H, Ghatei M, Adrian T, Bloom S, Dowling RH. Role of regulatory peptides in the small bowel and pancreatic adaptation of pancreatico-biliary diversion (PBD) following jejunal or ileal transposition in the rat. [Abstract]. Eur J Clin Invest 1982; 12: 27.

28 Morisset J, Benrezzak O. Polyamines and pancreatic growth induced by caerulein. Life Sci 1984; 35: 247180.

29 Baylin SB, Stevens SA, Shakir KMM. Association of diamine oxidase and ornithine decarboxylase with maturing cells in rapidly proliferating epithelium. Biochim Biophys Acta 1978; 541 : 415-9.

30 Porter CW, Dworaczyk C, Ganis B, Weiser MM. Polyamines and biosynthetic enzymes in the rat intestinal mucosa and the influence of methylglyoxal-bis (guanylhydrazone) Cancer Res 1980; 40: 2330-5.

31 Yang P, Luk GD. The localization of polyamine biosynthesis along the villus-crypt axis during small intestinal proliferation. Gastroenterology 1984; 86: 1304.

32 Luk GD. Essential role of polyamine metabolism in hepatic regeneration. Gastroenterology 1986; 90: 12617.

33 Luk GD, Abeloff MD, McCann PP, Sjoerdsma A, Baylin SB. Long-term maintenance therapy of established human small cell variant lung carcinoma implants in athymic mice with a cyclic regimen of difluoromethylornithine. Cancer Res 1986; 46: 1849-53.

34 Hosomi M, Stace N, Murphy GM, Dowling RH. Cellular regulation of adaptive intestinal mucosal hyperplasia: Effect of the polyamine synthesis blocker DFMO. Gastroenterology 1985; 88: 1715.

35 Stace NH, Hosomi M, Vaja S, Murphy GM and Dowling RH. Polyamine synthesis inhibitor, difluoromethylornithine (DMFO) does not prevent the pancreatic hyperplasia induced by pancreatico-biliary diversion (PBD) in the rat. [Abstract]. Gut 1984; 25: A1159.

36 Benrezzak O, Morisset J. Effects of alpha-difluoromethyl ornithine on pancreatic growth induced by caerulein. Regul Peptides 1984; 9: 143-53.

37 Morisset J, Benrezzak O. Reversal of alpha-difluoromethyl ornithine inhibition of caerulein-induced pancreatic growth by putrescine. Regul Peptides 1985; 11 : 201-8.

38 Pösö H, Pegg AE. Effect of alpha-difluoromethylornithine on polyamine and DNA synthesis in regenerating liver. Biochim Biophys Acta 1982; 696: 179-86.

39 Seidel ER, Tabata K, Dembinski AB, Johnson LR. Attenuation of trophic response to gastrin after inhibition of ornithine decarboxylase. Am J Physiol 1985; 249: G16-20. 